“드 2014 IEEE. Personal use of this material is permitted. Permission from IEEE must be obtained for all other uses, in any current or future media, including reprinting/republishing this material for advertising or promotional purposes, creating new collective works, for resale or redistribution to servers or lists, or reuse of any copyrighted component of this work in other works." 


\section{LTE-Advanced Fair Intelligent Admission Control - LTE-FIAC}

\author{
Fatima Furqan, \\ INEXT Centre for Innovation in IT \\ Services and Applications \\ University of Technology, Sydney \\ Fatima.furqan@ @student.uts.edu.au
}

\author{
Doan B. Hoang \\ INEXT Centre for Innovation in IT \\ Services and Applications \\ University of Technology, Sydney \\ Doan.Hoang@uts.edu.au
}

\author{
Iain B. Collings \\ ICT Centre, CSIRO, Australia. \\ iain.collings@csiro.au
}

\begin{abstract}
Radio Admission Control (RAC) is a key function of the Radio Resource Management (RRM) at layer 3 of an eNodeB; however, the 3GPP standard does not specify the RAC and is left as an eNodeB vendor specific. Most admission control schemes proposed to date do not ensure differentiation among the users at different priority levels. Also, they do not provide fairness among the users with the same priority. This paper proposes a novel RAC scheme for Long Term Evolution (LTEAdvanced) networks based on the combined idea of complete sharing and virtual partitioning. It introduces a step wise degradation scheme, to prioritize the high priority traffic in state of resource limitations.
\end{abstract}

A detailed and comprehensive simulation is performed in Opnet to show the efficiency of the proposed RAC scheme. The simulation results demonstrate that the proposed RAC scheme minimizes the call blocking probability and adheres extremely well to the bandwidth constraints of different traffic types.

Keywords: Admission Control; blocking probability; Fairness; Quality of Service (QoS).

\section{INTRODUCTION}

In Long Term Evolution (LTE) networks, the Evolved Packet System (EPS) bearer can be initiated by the User Equipment (UE) or by the packet core network. Radio Admission control (RAC), a key function of Radio Resource Management (RRM) at layer 3 of an eNodeB, decides to accept or reject the request for new EPS bearer. The Quality of Service (QoS) profile of each EPS bearer includes QoS Class Identifier (QCI) and Allocation and Retention priority (ARP). QCI defines the forwarding treatment of IP packets received on a bearer. In situations when resources are limited, RAC uses ARP to determine whether a new bearer can be established. RAC aims to achieve high resource utilization. However, it grants a request only when it estimates that the network can guarantee acceptable QoS to new and all active EPS bearers.

When the network resources are limited, the RAC can admit high priority connections using either of the two schemes: a) preemption scheme [1] ,or b) the degradation schemes [2,3]. Preemption scheme in [1] allows high priority bearer to remove the low priority bearers to obtain sufficient resources. It results in unfairness with the flows of low priority bearers. The degradation scheme in [2, 3] instantly degrades the rate allocated to low priority connections to their respective GBR even if only a single PRB is required. Thus, additional resources are added to the complete sharing based available resource pool and the RAC is free to allocate them to the new connection of any service type. In this way, in times of resource scarcity, resources that can be given to high priority class by applying degradation on low priority classes reduce. It results in unfair bandwidth allocation among different priority classes. Moreover, the degradation schemes in $[2,3]$ degrades connections at the same ARP, one by one until sufficient resources are obtained. In this way all the connections with same ARP may not be degraded and cause unfair bandwidth share to the flows at the same priority level.

To address the issues of unfair bandwidth allocation among different priority classes and among the flows at the same priority level, we propose a Fair Intelligent Admission Control scheme for the LTE advanced networks (LTE-FIAC). This scheme combines the idea of Complete Sharing (CS) and Virtual Portioning (VP) resource allocation schemes. To guarantee maximum resource utilization, it avoids any resource reservation. So, LTE-FIAC uses a variation of VP which does not reserve even nominal bandwidth for any traffic group. In state of limited channel resource availability, it employs step wise degradation scheme to provide differentiation among the flows of different service types. In addition, we introduce a variable size degradation step that allows different amount of degradation to be applied at various priority levels. LTE-FIAC degradation procedure provides fair resource allocation among connections at same/different priority levels.

The paper is organized as follows. Section II introduces the proposed RAC scheme. Section III presents the simulation setup and discusses the results of simulations. Finally, conclusion is given in section IV.

\section{PROPOSED SCHEME.}

In this section, we propose a new admission control algorithm namely Fair Intelligent Admission Control for the LTE Advanced systems (LTE-FIAC). The notations for the system level and the bearer level parameters that we use in this paper are as follows.

A. Notations.

Total_PRB $\mathrm{B}_{\mathrm{x}} \quad$ Total amount of bandwidth available for each direction ' $x$ ' at the eNodeB in terms of PRBs.

$\mathrm{PRB}_{\text {used }_{\mathrm{x}}}$ Total number of PRBs used in ' $\mathrm{x}$ ' direction.

PRB $_{\text {avail }_{\mathrm{x}}} \quad$ Total_PRB $_{\mathrm{x}}-\mathrm{PRB}_{\text {used }_{\mathrm{x}}}$

$\mathrm{PRB}_{\mathrm{REQ}_{\mathrm{X}}} \quad \mathrm{PRB}$ Requested by incoming connection.

$\mathrm{PRB}_{\text {REM }} \quad \mathrm{PRB}_{\mathrm{REM}}=\mathrm{PRB}_{\mathrm{REQ}_{\mathrm{x}}}-\mathrm{PRB}_{\mathrm{avai}_{\mathrm{x}}}\left(\right.$ If $\mathrm{PRB}_{\mathrm{REQ}}>\mathrm{PRB}_{\text {avail }_{\mathrm{x}}}$ Else $P_{R B} B_{\text {REM }}=0$ ) 


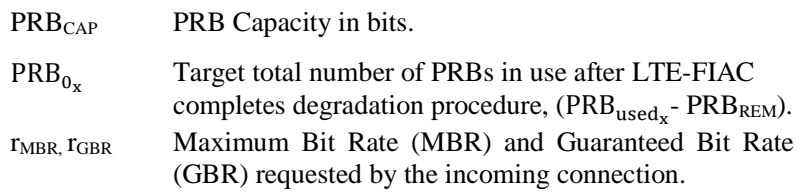

\section{B. Description of $C A C$}

In this section we describe our proposed LTE-FIAC. This scheme is based on the idea of $\overline{C S}$ and $\overline{V P}$. The RAC offers $\mathrm{CS}$, as multiclass users share the common pool of available resources. In situations when resources are scarce, it uses VP to differentiate among multiservice users. Consequently, to be able to accept an incoming request of high priority, it reduces resources allocated to low priority bearers. To apply degradation, it uses a step wise degradation scheme. In our proposed LTE-FIAC when a new connection request arrives with $\mathrm{PRB}_{\mathrm{REQ}_{\mathrm{x}}}$, LTE-FIAC first checks resource availability.

\section{1) No Degradation}

When the connection request arrives, LTE-FIAC estimates the resource availability in the network for both directions that is uplink (UL) and downlink (DL) as indicated by ' $x$ ' in the following equation.

$$
P R B_{R E Q_{x}}=\left\{\begin{array}{c}
P R B_{R E Q_{x}}^{M B R}, \sum_{i=0}^{N} \sum_{j=0}^{n_{i}} P R B_{j_{x}}+P R B_{R E Q_{x}}^{M B R} \leq \text { Total_PRB } \\
P R B_{R E Q_{x}}^{G B R}, \sum_{i=0}^{N} \sum_{j=0}^{n_{i}} P R B_{j_{x}}+P R B_{R E Q_{x}}^{G B R} \leq \text { Total_PRB }
\end{array}\right.
$$

In (1), ' $\mathrm{i}$ ' is the index of each QCI and ' $\mathrm{j}$ ' refers to each data flow within each QCI. PRB $\mathrm{j}_{\mathrm{x}}$ is the total number of PRBs assigned per second to a bearer $\mathrm{j}$ in direction ' $\mathrm{X}$ ' to meet its MBR or GBR. It set the connection status to ready to admit, if enough resources are available in the network to meet either the MBR or GBR of the incoming connection.

\section{2) Degradation}

In situations, when enough resources are not available in the network to admit the connection even with its GBR, LTEFIAC admit high priority connections using the step wise degradation. Following equation is applied to validate that enough resources can be obtained by degrading the rate allocated to the connections of low priority.

$$
P R B_{R E Q_{X}}=\left\{\begin{array}{c}
P R B_{R E Q_{x^{\prime}}}^{G B R}\left[\sum_{i=0}^{N} \sum_{j=0}^{n_{i}} P R B_{j_{x}}-\left[f(j, u)\left(P R B_{j_{x}}-P R B_{j_{x}}^{G B R}\right)\right]\right] \\
+P R B_{R E Q_{x}}^{G B R} \leq \text { Total_PRB } B_{x} \\
0, \quad \text { otherwise }
\end{array}\right.
$$

In (2), ' $u$ ' is the ARP index of the incoming connection and ' $f(j, u)$ ' is a function given in [1]. Function $f(j, u)$ returns 1 only if incoming bearer has lower degradation priority than the bearer ' $\mathrm{j}$ '. Otherwise, it returns 0 . Degradation priority of bearers is based on the associated ARP value. Equation (2) shows that during degradation, the rate allocated to the connections of low priority can be reduced to their respective GBR only. Also, degradation is applied only to meet the GBR of the incoming connection. Using (2), if LTE-FIAC estimates that adequate resources can be obtained for both directions, by degrading the connections of low priority, it sets the connection status to ready to admit. Otherwise, LTE-FIAC rejects the incoming request.

\section{3) Step Wise Degradation}

When LTE-FIAC in (2) estimates that to admit incoming connection enough resources can be obtained from low priority connections, it applies step wise degradation scheme. To apply stepwise degradation, LTE-FIAC uses an intelligent function of PRBs which is as follows.

$$
f(P R B s)=1-\frac{\text { Total_PRB }_{x}-P R B_{\text {used }}}{\text { Total_PRB }_{-}-P R B_{0_{x}}}
$$

In (3), $\mathrm{PRB}_{\text {used }_{\mathrm{x}}}$ is the total number of PRBs occupied by the existing connections. $\mathrm{PRB}_{0_{\mathrm{x}}}$ is the target number of used PRBs after degradation process completes. The step size is calculated as follows.

$$
\delta=\mathrm{f}(\mathrm{PRBs}) * \mathrm{PRB}_{\mathrm{CAP}}
$$

In (4), $\delta$ is the degradation step size. So, LTE-FIAC degrades the rate allocated to all connections at a specific ARP equal to a fraction of a PRB at a time. The key feature of the proposed step size is that it maintains fair share of bandwidth among different priority classes. As when degradation is first applied on the connections of the lowest priority ARPs, the number of used PRBs $\left(\operatorname{PRB}_{\text {used }_{\mathrm{x}}}\right)$ and also the value of $\mathrm{f}(\mathrm{PRBs})$ reduce. It in turn reduces the $\delta$ and results in less degradation applied on the connections with higher priority ARPs. So, variable size degradation step allows different amount of degradation to be applied at various priority levels.

\section{LTE-FIAC degradation scheme follows following rules.}

1. Degradation is applied only to provide the GBR of the incoming connection.

2. Degradation is applied only on the flows with low priority ARP starting with the lowest priority ARP.

3. When executing degradation, a connection bandwidth can be reduced only to its GBR.

The detailed steps of degradation procedure are as follows.

Step 1: The degradation procedure first sorts the admitted connections in a degradation priority descending order stated by ARP. Step 2: The degradation procedure starts from the lowest priority ARP. Step 3: MBR of all data flows at the lowest priority ARP is degraded uniformly, equal to the step size (4). Consequently, step 3 enables LTE-FIAC to ensure fairness among the data flows with same ARP. The LTE-FIAC applies degradation on the MBR of data flows and keeps reducing it until either enough resources are obtained or the MBR of data flows reduces to GBR. Based on the degradation applied, LTE-FIAC updates the used PRBs $\left(\mathrm{PRB}_{\text {used }_{\mathrm{x}}}\right)$ and the available PRBs $\left(\mathrm{PRB}_{\text {avail }_{\mathrm{x}}}\right)$. Step 4: When MBR of all data flows at a specific ARP becomes equal to their respective GBR, LTE-FIAC moves to the next lowest priority ARP. Step 5: It continues degradation process and keeps moving to the next lowest priority levels until either sufficient resources are obtained or the next lowest priority ARP is equal to the ARP of the incoming connection. Step 5 enables LTE-FIAC to ensure the fair share of bandwidth among different priority classes. As in this step, LTE-FIAC degrades the bandwidth equals to exactly what is required. Hence, it retains the 
resources with low priority connections to admit incoming high priority connections.

\section{PERFORMANCE EVAluAtion}

The overall goal of the simulation is to investigate the effectiveness of the proposed scheme in terms of call blocking probability and fairness among service flows at same/ different priority levels. The performance of LTE-FIAC is compared with a tuned reference admission control algorithm per TTI $[4,5]$, stated as follow.

$$
\sum_{i=1}^{K} N_{i}+N_{\text {new }} \leq N_{\text {total }}
$$

Where, $\mathrm{N}_{\text {total }}$ is the total number of PRBs in the network per second. $\mathrm{K}$ is the number of existing connections in the cell. $\mathrm{N}_{\mathrm{i}}$ represents the number of PRBs required per second by the active connection ' $i$ ' to satisfy its MBR. The reference AC, hereafter referred as Ref Scheme, grants a request if the sum of PRBs requested by the new $\left(\mathrm{N}_{\text {new }}\right)$ and the active connections is less than or equal to $\mathrm{N}_{\text {total }}$.

We also compared LTE-FIAC degradation procedure with the degradation process (referred here as Ref-Deg) proposed in [3]. They are similar in terms of applying degradation on the connections of low priority, but Ref-Deg scheme degrades the connections with a degradation level of $r_{\mathrm{MBR}}-\mathrm{r}_{\mathrm{GBR}}$.

\section{A. Simulation Setup}

The simulations are performed using the LTE module in system level simulator, Optimized Network Engineering Tool (OPNET) release 17.1.A. In the current simulation setup, eNodeB operates in FDD mode and employs bandwidth of 3 $\mathrm{MHz}$. For simplicity, the simulation model consists of a single cell based on 3GPP LTE system model. The requests arrivals in the system are modeled by a Poisson process. The simulation includes three service classes (voice, video and web). The arrival rate of connections of each service type is set to be same that is $\lambda_{\text {voice }}=\lambda_{\text {video }}=\lambda_{\text {web }}$. The calls from the same service class have same QCI and ARP values, thus same priority level. The detailed traffic model settings are given in Table I.

TABLE I. QOS REQUIREMENTS

\begin{tabular}{|c|c|c|c|c|c|}
\hline Services & QCI & $\begin{array}{l}\text { ARP- } \\
\text { Priority }\end{array}$ & $\begin{array}{l}\text { Delay } \\
\text { Budget }\end{array}$ & $\begin{array}{l}\text { MBR } \\
\text { (kbps) }\end{array}$ & $\begin{array}{l}\text { GBR } \\
\text { (kbps) }\end{array}$ \\
\hline Voice & 1 & 1 & $100 \mathrm{~ms}$ & 68 & 68 \\
\hline Video & 4 & 5 & $150 \mathrm{~ms}$ & 256 & 96 \\
\hline Web & 8 & 4 & $300 \mathrm{~ms}$ & 128 & 32 \\
\hline
\end{tabular}

Voice UEs transmits using the VoIP G.711. The video service users transmit $256 \mathrm{kbps}$ H.263 video streams. The trace file for the $256 \mathrm{kbps} \mathrm{H.263} \mathrm{encoded} \mathrm{Jurassic} \mathrm{Park} \mathrm{movie}$ is obtained from the website in [6]. The total simulation time is 300 seconds.

\section{B. Simulation Results}

This section presents and discusses the results of our simulations to show the performance of our proposed LTEFIAC.

\section{1) Call Blocking Probability (BP)}

Figs. 2, 3 and 4 present the connection blocking probability as a function of call arrival rate. From them we can conclude that as the connection arrival rate increases the $\mathrm{BP}$ also increases. Fig. 1 shows the connection blocking probability for the Ref scheme given in (5).

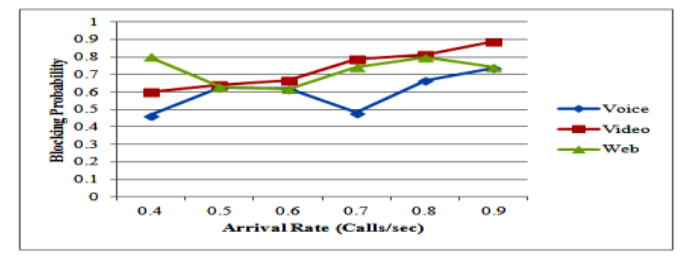

Fig. 1. BP of connections at the eNodeB with Ref Scheme

Fig. 1 shows the BP of voice traffic is less as compared to web and video traffic, but the BP of video traffic is higher than web traffic. This is because the bandwidth demand of video traffic is much higher than web traffic, so the system accommodates more web connections as compared to video traffic. Hence, Ref scheme cannot guarantee the differentiation among different priority classes.

Fig. 2 show the BP for the Ref-Deg scheme [3]. Fig. 2 shows the BP of voice traffic is less as compared to web and video traffic, but as the arrival rate increases the BP of voice traffic becomes even higher than web traffic. Additionally, the $\mathrm{BP}$ of video is quite higher than web traffic. This is because in order to admit a connection of high priority, it degrades the rate of lower priority connections directly to their GBR, until sufficient resources are obtained. So, when degradation procedure completes, the network acquires quite additional resources in its available resource pool than requested by the incoming connection. The available resource pool is based on complete sharing hence the additional resources can be assigned to the incoming connection of any service type. Consequently, ref-Deg scheme also can not ensure fair bandwidth allocation among the different priority classes.

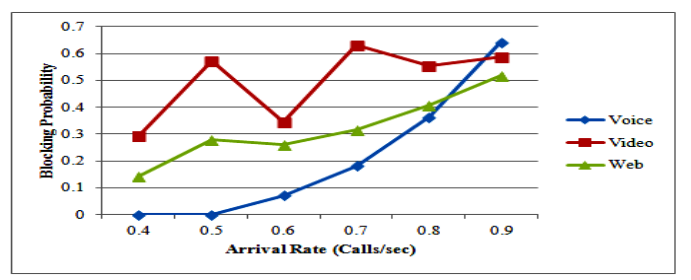

Fig. 2. BP of connections at the eNodeB with Ref-Deg Scheme

Fig. 3 shows the BP for the LTE-FIAC scheme. The BP for voice traffic is minimum for LTE-FIAC scheme as compared to the Ref scheme in (5) and the Ref-Deg scheme in [3]. The BP of video is overall lower compared to web traffic. So LTE-FIAC ensures service differentiation which can be contributed to the fact that in order to admit a connection of high priority, LTE-FIAC step wise degrades the connections of lower priority only until enough resources are obtained. So, when LTE-FIAC performs degradation, the complete sharing based available resource pool becomes a little higher than the resources requested by the incoming connection. Now as the virtual partitioning is applied to prioritize the high priority class, so in future when a high priority connection arrives, LTE-FIAC will be able to admit it by degrading the lower priority connections. In this way, LTE-FIAC scheme by 
degrading the lower priority connections to only what is exactly required, holds the resources for the incoming high priority connections and guarantees the service differentiation.

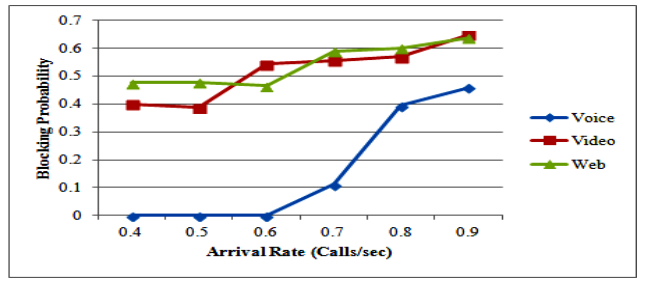

Fig. 3. BP of connections at the eNodeB with LTE-FIAC

2) Fairness among flows of Different priority classes

Fig. 4 shows the complete distribution of the number of granted requests for each type of service. For LTE-FIAC the number of granted requests is in the order of priority that is VoIP > Video > Web. Whereas for the Ref-Deg scheme, the priority distribution of the number of granted requests is random. Fig.4 shows for Ref-Deg scheme the admitted requests of high priority video are very less than the web (also refer to Fig. 2). So, when call arrival rate is high, even though LTE-FIAC admits fewer connections as compared to Ref_Deg scheme but it achieves fair distribution of bandwidth among different priority classes than the Ref-Deg scheme.

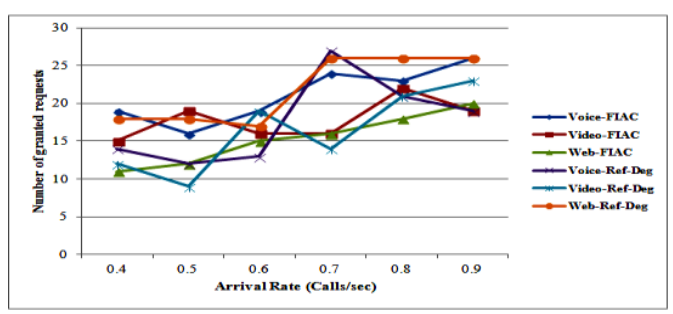

Fig . 4. Number of granted requests for each traffic

3) Fairness among flows within same priority class

In times when resources are scarce in the network, the RefDeg scheme one by one degrades the connections with lowest priority ARP with a degradation level of $r_{M B R}-r_{G B R}$. When enough resources are obtained, it stops degradation procedure. This type of degradation leads to unfairness among the connections at the same ARP level. Fig. 5 (a) shows that at around 204 sec of simulation, connections with high priority ARP arrives. To obtain sufficient resources, it degrades only video connections v-6 and v-7. So, the throughputs of v-6 and $\mathrm{v}-7$ reduced to their respective GBR. Whereas, the throughputs of other video connections $v-1 \ldots v-5$ at the same ARP level with similar values of MBR and GBR parameters, are still at their respective MBR. This leads to unfair resource allocation to the connections at the same priority level. So as Ref-Deg scheme cannot ensure fair bandwidth share among the connections at same priority level.

Fig. 5 (b) shows LTE-FIAC degrades all the connections with low priority ARP with a degradation step size of (4). Once enough resources are obtained, it stops degrading the connections. This type of degradation leads to fairness among the connections at the same ARP level. Fig. 5(b) shows that at around $204 \mathrm{sec}$ of simulation, when voice connections arrive with high priority ARP, LTE-FIAC degrades all video flows equally. So all video connections v-1...v-7 at the same ARP and with similar values of MBR and GBR, have the throughputs at closely the same rate. So, LTE-FIAC provides fair share of bandwidth to the connection at same ARP.

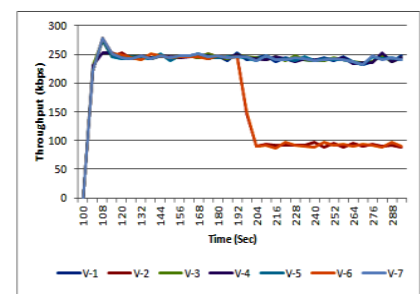

(a)

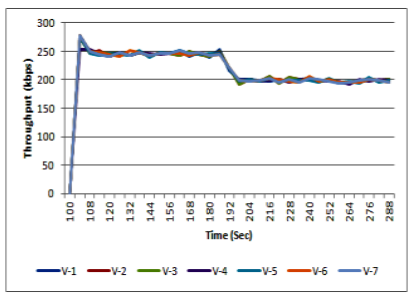

(b)
Fig. 5. Total Throughput (kbps) of video bearers (a) with Ref-Deg (b) With LTE-FIAC

In short, LTE-FIAC achieves lower blocking probability and maintains priority among connections of different traffic types. LTE-FIAC also ensures fairness among service data flows at same priority during the degradation procedure.

\section{CONCLUSION}

In this paper we presented LTE-FIAC, an intelligent admission control scheme. By using simulations, we evaluated the performance of LTE-FIAC scheme. The results showed that LTE-FIAC achieves lower blocking probability, and guarantees fair share of bandwidth among service flows at same/different priority levels. The current implementation of LTE-FIAC takes only the incoming user's QoS requirements and the channel conditions at the connection's arrival time into account. Whereas, during the lifetime of the connection the channel conditions may degrade that adversely affect the QoS of the connection. So, our future research aims to modify LTE-FIAC to enable it to deal with the change in the user demand caused by the channel fluctuations. Moreover, we aim to implement LTE-FIAC with the load estimation module. The load estimation module detects the congestion at the core side of the LTE networks by measuring the queue length at the output buffer of the eNodeB. LTE-FIAC with load estimation module ensures that the incoming connection will not overload the output buffer of the eNodeB and preserves the QoS of existing traffic flows in a fair manner.

\section{REFERENCES}

[1] R. Kwan, Arnott. R, Trivisonno. R, Kubota. M., "On Pre-Emption and Congestion Control for LTE Systems," in IEEE 72nd Vehicular Technology Conference Fall (VTC'10), Fall 2010, pp. 1-5.

[2] S. V. Priya and J. V. Franklin, "Extensive DBA-CAC mechanism for maximizing efficiency in 3GPP: LTE networks," in International Conference on Recent Advances in Computing and Software Systems (RACSS'12), 2012, pp. 233-237.

[3] Q. Manli, et al., "A Novel Radio Admission Control Scheme for Multiclass Services in LTE Systems," in Global Telecommunications Conference, (GLOBECOM'09), 2009, pp. 1-6.

[4] M. Anas, et al., "QoS-Aware Single Cell Admission Control for UTRAN LTE Uplink," presented at the IEEE Vehicular Technology Conference, (VTC'08), Spring 2008

[5] M. Anas, Rosa. C , Calabrese. F. D, Pedersen.K.I, Mogensen.P.E "Combined Admission Control and Scheduling for QoS Differentiation in LTE Uplink," presented at the IEEE $68^{\text {th }}$ Vehicular Technology Conference, (VTC'08), Fall 2008.

[6] http://www.opnet.com (opnet modeler release 17.1.A)

[7] H.263 Video Traces. 7/7/2013). Available: http://www2.tkn.tuberlin.de/research/trace/ltvt.html 